\title{
Peripheral Neuroblastic Tumours
}

\author{
Jasila Akbar $^{1}$, Prema Saldanha ${ }^{2}$ \\ ${ }^{1}$ Post Graduate, Department of Pathology, ${ }^{2}$ Professor, Department of Pathology, \\ Yenepoya Medical College, 575018, Mangalore, Karnataka, India \\ *Corresponding author - Jasila Akbar, drjasila@gmail.com
}

Received 01 February 2019;

Accepted 22 February 2019;

Published 24 February 2019

\begin{abstract}
Background: Peripheral neuroblastic tumours arise from the neural crest cells in the sympathetic nervous tissue. These are rare, but are the most common among the extracranial solid tumours occurring in children. Method: This was a retrospective study conducted on 15 cases of peripheral neuroblastic tumours. The demographic details were recorded and the slides were analysed for the microscopic features and classified according to International Neuroblastoma Pathology Classification (INPC). Results: Out of the 15 peripheral neuroblastic tumour cases, 8 were males and 7 females. Seven cases came under age group <1.5years, 5 were between 1.5 years to 5 years and 5 cases were over 5 years of age. Seven tumours were from the retroperitoneal region, 4 cases were from the adrenal gland, 2 from intracranium and one case each was from the neck and the mediastinal regions. There were 8 Neuroblastoma cases, 2 cases of Ganglioneuroblastoma and 5 cases of Ganglioneuroma. It was found that 8 tumours were in the favourable histology group and 7 tumours in the unfavourable histology group. Conclusion: Peripheral neuroblastic tumours are rare. It is important to classify them accurately according to the various categories which help to determine the prognosis.
\end{abstract}

Keywords: Peripheral neuroblastic tumours, Neuroblastoma, Ganglioneuroma, Ganglioneuroblastoma

\section{Introduction}

Peripheral neuroblastic tumours are derived from precursor cells of the sympathetic nervous system. These cells are of sympathoadrenal lineage and migrate from the neural crest to form the sympathetic ganglia, chromaffin cells of the adrenal medulla, and the paraganglia; thus they can develop anywhere along the distribution of the sympathoadrenal neuroendocrine system. Peripheral neuroblastic tumours represent a spectrum of maturation ranging from the most primitive neuroblastoma(NB) to the most mature ganglioneuroma(GN) with ganglioneuroblastoma(GNB) being in an intermediate stage of maturation. ${ }^{[1,2]}$

Over $80 \%$ are detected in patients under the age of 4 years, and the median age at diagnosis is 21 months. The most common sites of origin of neuroblastic tumours are the adrenal region, followed by extraadrenal sites like retroperitoneum and chest. These tumours are known to show unique and often unpredictable clinical behaviour. ${ }^{[3]}$

The study was undertaken to review the features of peripheral neuroblastic tumour cases seen at our institution during the last 8 years.

\section{Method}

This was a retrospective study conducted on 15 cases of peripheral neuroblastic tumours which were retrieved from the archives of the Department of Pathology. The clinical data was obtained from the records. The slides were analysed for the histopathological features.

The tumours were classified according to the International Neuroblastoma Pathology Classification (INPC). They were categorized into two prognostic groups- Favourable and Unfavourable histology using the Shimada system. ${ }^{[1-4]}$

\section{Results}

Out of the 15 peripheral neuroblastic tumour cases, 8 were males and 7 females. Seven cases came under age group <1.5years, 5 were between 1.5 years to 5 years and 5 cases were over 5 years of age. Seven tumours were from the retroperitoneal region, 4 cases were from the adrenal gland, two intracranial tumours and one case each was from the neck and the mediastinal regions.

Eight cases were classified as Neuroblastoma, two cases were Ganglioneuroblastoma intermixed. There were no Ganglioneuroblastoma,nodular cases. Five cases were categorised as Ganglioneuroma. The details of the cases studied are given in Table- 1 . The gross and the microscopic appearances are shown in Figures 1 to 3 . 
Table 1: Details of cases in our study

\begin{tabular}{cccccc}
\hline Serial & Age (years) & Sex & Site & Diagnosis & Prognostic Group \\
\hline $\mathbf{1}$ & 5 & $\mathrm{~F}$ & Intracranial & Neuroblastoma - Differentiating & Unfavourable \\
$\mathbf{2}$ & 0.5 & $\mathrm{~F}$ & Retroperitoneum & Neuroblastoma- Undifferentiated & Unfavourable \\
$\mathbf{3}$ & 4 & $\mathrm{M}$ & Adrenal & Neuroblastoma- Undifferentiated & Unfavourable \\
$\mathbf{4}$ & 1.5 & $\mathrm{M}$ & Adrenal & Neuroblastoma-Poorly differentiated & Unfavourable \\
$\mathbf{5}$ & 2 & $\mathrm{~F}$ & Retroperitoneum & Neuroblastoma-Poorly differentiated & Unfavourable \\
$\mathbf{6}$ & 4 & $\mathrm{M}$ & Retroperitoneum & Neuroblastoma-Poorly differentiated & Unfavourable \\
$\mathbf{7}$ & 30 & $\mathrm{M}$ & Intracranial & Neuroblastoma-Poorly differentiated & Unfavourable \\
$\mathbf{8}$ & 1 & $\mathrm{~F}$ & Retroperitoneum & Neuroblastoma-Poorly differentiated & Favourable \\
$\mathbf{9}$ & 2.5 & $\mathrm{~F}$ & Neck & Ganglioneuroblastoma & Favourable \\
$\mathbf{1 0}$ & 19 & $\mathrm{~F}$ & Mediastinum & Ganglioneuroblastoma & Favourable \\
$\mathbf{1 1}$ & 28 & $\mathrm{M}$ & Retroperitoneum & Ganglioneuroma- Mature & Favourable \\
$\mathbf{1 2}$ & 3 & $\mathrm{M}$ & Retroperitoneum & Ganglioneuroma- Mature & Favourable \\
$\mathbf{1 3}$ & 10 & $\mathrm{~F}$ & Retroperitoneum & Ganglioneuroma- Mature & Favourable \\
$\mathbf{1 4}$ & 30 & $\mathrm{M}$ & Adrenal & Ganglioneuroma- Maturing & Favourable \\
$\mathbf{1 5}$ & 9 & $\mathrm{M}$ & Adrenal & Ganglioneuroma- Mature & Favourable \\
\hline
\end{tabular}

Out of the eight neuroblastoma cases 4 were in the 1.5 to 5 years

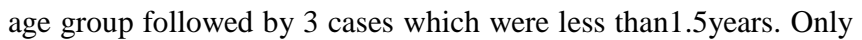
a single case was more than 5 years of age. An equal gender distribution was noted in these cases. Retroperitoneum was the most common site for neuroblastoma (4 out of 8 ) followed by two each adrenal and intracranial tumours. Based on the grade of neuroblastic differentiation of the given tumours, 2 cases were Undifferentiated, five cases were Poorly differentiated and a single case was diagnosed as Differentiating neuroblastoma. All the neuroblastomas except a single case were categorised as Unfavourable histology. The case with favourable histology was poorly differentiated, with age 1year and low MKI. Both

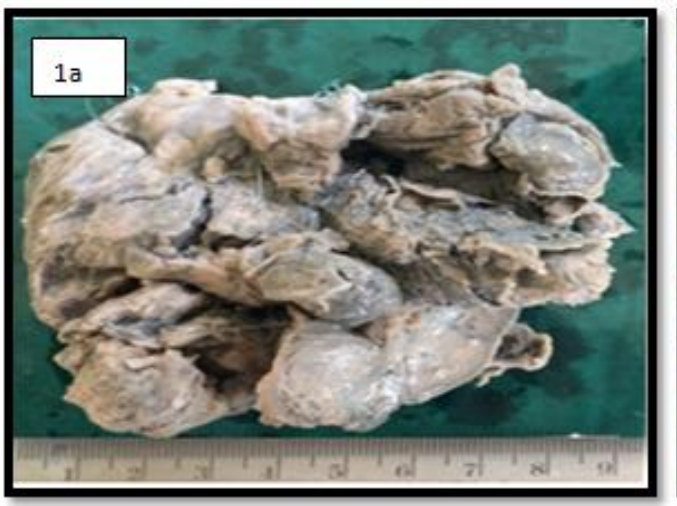

ganglioneuroblastoma cases were categorised as Favourable histology group.

Majority of the ganglioneuroma cases (4 out of 5) were more than 5 years. Most of cases were males ( 3 out of 5). Retroperitoneum was the most common site ( 3 out of 5) followed by adrenal tumours. Out of these 4 were Mature ganglioneuroma which had Schwann cell stroma and scattered mature ganglion cells with satellite cells. A single case was categorised as Maturing ganglioneuroma which had Schwann cell stroma with scattered maturing ganglion cells without satellite cells or neuropil. All the ganglioneuroma cases were categorised as Favourable Histology group.

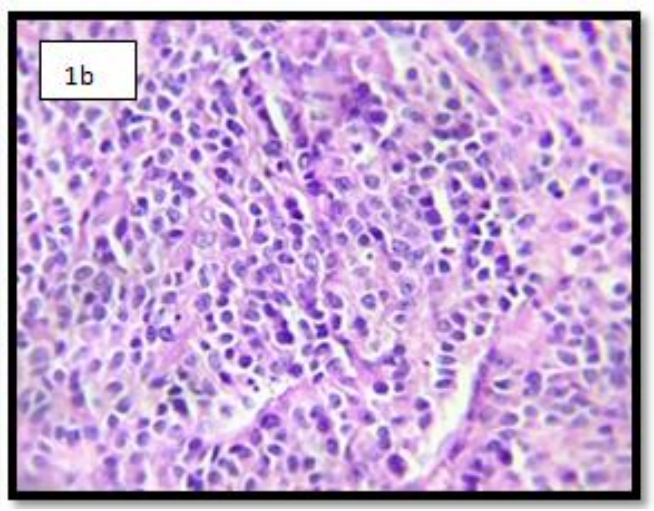

Figure 1a: Retroperitoneal neuroblastoma, cut surface nodular, grey brown to dark brown. Figure 1b: Neuroblastoma composed of closely packed tumor cells without identifiable rosettes or neurofibrillary matrix, no evidence of ganglion cell differentiation (H\&E x40).
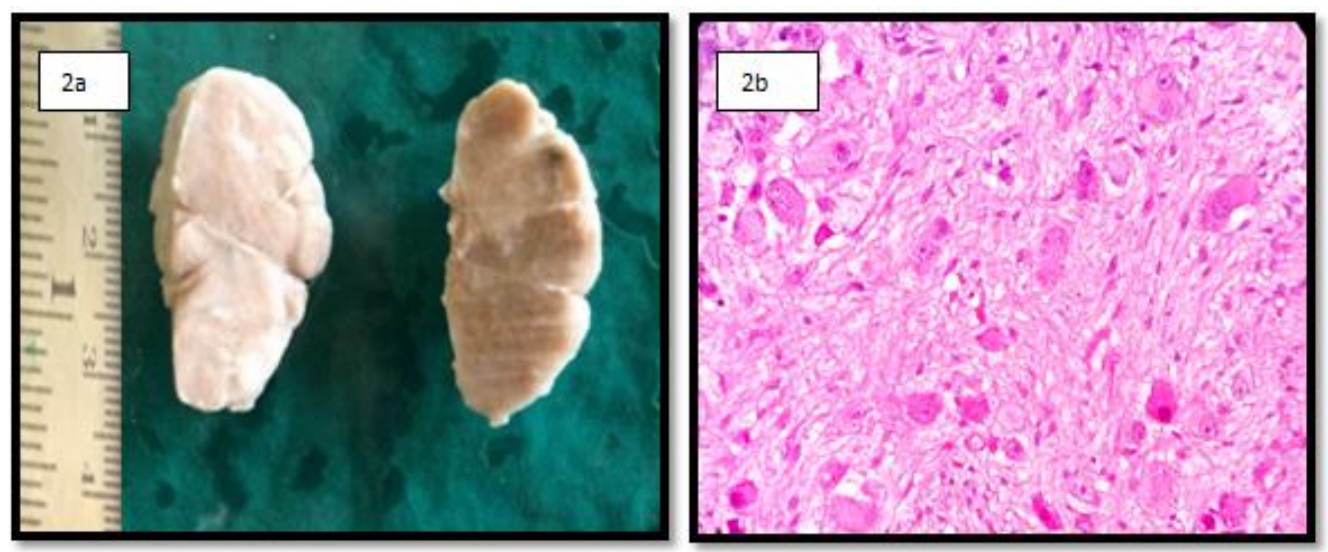

Figure 2a: Ganglioneuroblastoma-neck, cut surface is grey white glistening, Figure 2b: Ganglioneuroblastoma with mature ganglion cells, fibrillary stroma and small round neuroblastic cells(H\&E x40) 


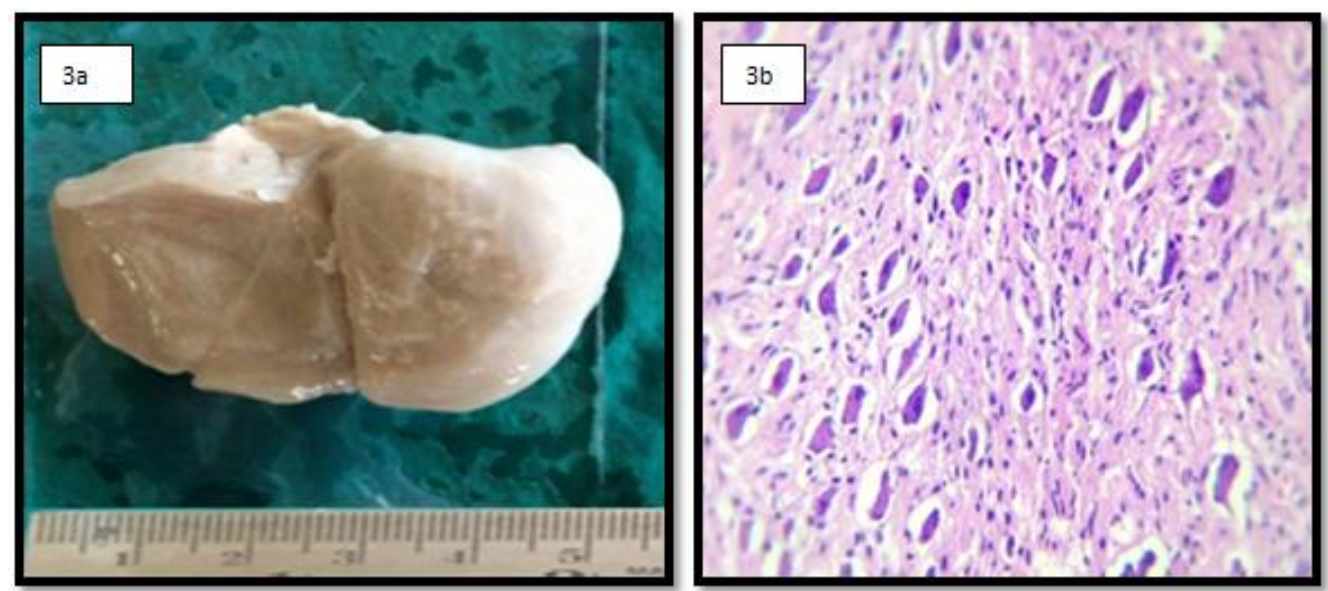

Figure 3a: Retroperitoneal Ganglioneuroma, Cut surface grey white nodular and mucoid. Figure 3b: Ganglioneuroma- shows mature ganglion cells surrounded by a Schwann cell-rich stroma (H\&E x40)

\section{Discussion}

Peripheral neuroblastic tumours have been divided traditionally into three categories: neuroblastoma (NB), ganglioneuroblastoma (GNB), and ganglioneuroma (GN). These categories have been considered as a continuum from the most immature to the most mature forms of Neuroblastic tumours. ${ }^{[5,6]}$

According to the International Neuroblastoma Pathology Classification of peripheral neuroblastic tumours, the individual tumours were classified into four categories: 1) Neuroblastoma (Schwannian stroma poor) - NB, 2) Ganglioneuroblastoma, intermixed (Schwannian stroma-rich) - GNBi, 3) Ganglioneuroma (Schwannian stroma-dominant) - GN, 4) Ganglioneuroblastoma, nodular (composite, Schwannianstroma-rich/stroma-dominant and stroma-poor) - GNBn. The tumours in the neuroblastoma category were further divided into three subtypes - undifferentiated, poorly differentiated, and differentiating-based on the grade of neuroblastic differentiation. The tumours in the ganglioneuroma category were classified into two subsets: maturing and mature. ${ }^{[1-4]}$

Neuroblastoma (NB) is the most common extracranial solid tumour in childhood. It is a malignancy arising within the neuronal ganglia of the peripheral sympathetic nervous system. These neuronal structures originate from the ventrolateral neural crest cells that migrate away from the neural tube early during embryogenesis. Thirty percent of neuroblastoma tumours arise within the adrenal medulla, approximately $60 \%$ will arise from abdominal paraspinal ganglia, and the remaining from the sympathetic ganglia in the chest, head and neck, and pelvis. The clinical presentation and outcome of neuroblastoma are highly variable. Long-term survival is dependent on the age of the patient and degree of differentiation. The prognosis of patients exhibiting more primitive crest-like tumours will be worse than patients with more differentiated tumours. ${ }^{[5]}$

Neuroblastomas are neuroblastic, Schwannian stroma-poor tumours and are composed of small, round primitive-appearing cells with dark nuclei, scant cytoplasm and poorly defined cell borders growing in solid sheets, ill-defined lobular or nesting pattern. Nuclear chromatin is finely dispersed in small clumps, imparting a "salt-and-pepper" appearance; nucleoli are mostly inconspicuous. In some tumours, the mitotic activity, pleomorphism and nuclear breakdown ("karyorrhexis") are prominent. The background often shows a faintly eosinophilic fibrillary material (neuropil) that corresponds to the neuritic processes of the primitive neuroblasts. Typically Homer-Wright pseudorosettes can be seen in which the tumour cells are concentrically arranged about a central space filled with neuropil. ${ }^{[1,7]}$

Based on the grade of neuroblastic differentiation, neuroblastomas are further divided into three subtypes - undifferentiated, poorly differentiated, and differentiating. Neuroblastoma- undifferentiated subtype, the tumour cells are undifferentiated and an identifiable background neuropil is absent. Neuroblastoma- poorly differentiated subtype is a tumour with a background of neuropil. The extent of neuropil formation may vary in the same tumour or from one tumour to other. Neuroblastoma, differentiating subtype usually has abundant neuropil, with $5 \%$ or more of the tumour cells showing differentiation toward ganglion cells. ${ }^{[1,2]}$

Based on the age-linked framework of the Shimada system the tumours were categorised into two prognostic groups: favourable histology group (FH) and unfavourable histology group (UH) which taken into consideration not only the age but also histologic features (grade of neuroblastic differentiation and mitosiskaryorrhexis index $).{ }^{[4]}$

The mitosis-karyorrhexis index (MKI) is defined as the number of tumour cells in mitosis and in the process of karyorrhexis. The three classes are 1) Low- < $2 \% \quad(100 / 5000$ mitotic and karyorrhectic cells) 2) Intermediate- 2-4\% (100-200/5000 mitotic and karyorrhectic cells) 3) High- > 4\% (>200/5000 mitotic and karyorrhectic cells).

The Favourable histology group consisting of 1) Poorly differentiated or differentiating subtype tumours with low or intermediate MKI $(<1.5$ years $)$ and 2) Differentiating subtype tumours with low or intermediate $\mathrm{MKI}(<1.5$ years $)$ or low MKI (1.5 years to 5 years).

The Unfavourable histology subgroup includes 1) Undifferentiated subtype tumours (any age), 2) Poorly differentiated subtype tumours (>1.5 years),3) High MKI (any age), 4) Intermediate MKI $(>1.5$ years),5) All neuroblastoma tumours with age $>5$ years having any grade of neuroblastic differentiation and any class of MKI.

Ganglioneuroblastoma nodular cases categorise as per neuroblastoma component in nodule(s). Regardless of the patient's 
age, tumours in the ganglioneuroblastoma intermixed category and the ganglioneuroma category are classified into the favourable histology group. ${ }^{[1-4]}$

Hiroyuki Shimada et al evaluated 746 neuroblastic tumours in 2001 and found 630 NB tumours,30 GNB tumours,10 GN tumours and 76 GNB nodular tumours. Their study confirmed the prognostic significance of the International Classification, substantiated age-linked prognostic effects of the morphologic indicators for patients with the tumours in the NB category, and supported the concept of an age-appropriate framework of maturation for patients with the tumours in the FH group. ${ }^{[24]}$

A low MKI indicated a good prognosis in patients up to age 5 years at the time of diagnosis, and an intermediate MKI indicated good prognosis in patients up to age 1.5 years at the time of diagnosis, whereas a high MKI was linked to a poor prognosis in any age. A more precise treatment planning will be possible with prior risk categorisation of patient according to age, stage, histopathology, MYCN oncogene status and other genetic aberrations. $^{[6,8,9]}$

Ganglioneuroblastomas contain primitive neuroblast and mature ganglion cells. They are considered to have an intermediate potential for malignancy as compared to benign ganglioneuroma and malignant neuroblastoma. ${ }^{[7,8]}$ Ganglioneuroblastomas are seen more often in adolescents, and are usually less aggressive than neuroblastomas. Approximately half of them are asymptomatic. $^{[9,10]}$ The most common sites of origin of ganglioneuroblastoma are the adrenal medulla followed by the extraadrenal retroperitoneum and the posterior mediastinum; neck and pelvis are less common sites. ${ }^{[11]}$ Other unusual locations are the thymus, lung, kidney, anterior mediastinum, stomach, and cauda equina. ${ }^{[12,13,14]}$ The most common presentation of ganglioneuroblastoma is abdominal distension, pain and neurologic symptoms due to nerve root compression and Horner's syndrome. ${ }^{[15,16,17]}$

Ganglioneuroblastoma has more than 50\% Schwann cells with randomly distributed neuroblast nests, maturing and mature ganglion cells and neuropil with nests of naked neuropil. Ganglion cell differentiation is shown by nuclear and cytoplasmic enlargement, distinct cell borders, and increased cytoplasmic eosinophilia. Nuclei may be eccentrically situated with margination of chromatin, and a prominent nucleolus may be seen. Ganglioneuroblastoma nodular is a composite tumour of different clones, consisting of either ganglioneuroma or ganglioneuroblastoma intermixed with one or more discrete expansile nodules of neuroblastoma. ${ }^{[16,18,19]}$

Ganglioneuroma (GN) is a rare, benign, differentiated neurogenic tumour that arises mainly from primordial neural crest cells and consists of mature Schwann cells, ganglion cells and nerve fibres. GNs may arise primarily or from neuroblastomas. The maturation of neuroblastoma into GN can either occur spontaneously or as a result of therapy. The posterior mediastinum and retroperitoneum are the two most common locations for GN, including $\mathrm{GN}$ in the adrenal medulla, estimated to represent about $20 \%$ of reported cases. ${ }^{[20,21]}$ GNs of adrenal origin occur most commonly in children and young adults. Diagnosis of GN is most commonly incidental. If the tumour reaches a considerable size, it may trigger shortness of breath, abdominal pain or chronic cough, depending on its location as a result of local compression. ${ }^{[22]}$
Ganglioneuroma, maturing subtype is composed predominantly of ganglioneuromatous stroma with a minor component of scattered, evenly or unevenly distributed collections of differentiating neuroblast and/or maturing ganglion cells in addition to fully mature ganglion cells. The individual neuroblastic cells merge into the predominantly ganglioneuromatous stroma. Ganglioneuroma, mature subtype is composed of mature Schwannian stroma and ganglion cells. The characteristic of these tumours are the fascicular pattern of neuritic processes accompanied by Schwann cells and perineurial cells. The mature ganglion cells are usually surrounded by satellite cells. The absence of a neuroblastomatous component is required complete maturation. ${ }^{[21,22,23,24]}$

\section{Conclusion}

From our study we observed that even though peripheral neuroblastic tumours are characteristically seen in infants and children, they can also present in adults, particularly ganglioneuromas. There was no sex predilection in the distribution of the tumours. The most common site of the tumour was retroperitoneum followed by adrenal for both neuroblastoma and ganglioneuroma.

The peculiarity of these tumours lies in their unpredictable biological evolution including the possibility of spontaneous or treatment-induced differentiation of neuroblasts into mature ganglion cells and the variability of their clinical and prognostic behaviour, which often reflect their degree of differentiation. Histopathological and prognostic classifications are very useful tools for distinguishing the patients who require aggressive treatment protocols from those who need no or reduced treatment modalities.

\section{References}

[1] Fletcher Ellis OI, Lee HSA, Pinder ES, Rakha EA. Tumors of the Autonomic Nervous System, Including Paraganglia. In: Fletcher C D M. Diagnostic histopathology of tumours. 4th ed. China. Elsevier; 2013.p. 2074-82.

[2] Mills S, Sternberg S. The Adrenal Glands. In: Sternberg's Diagnostic Surgical Pathology. 6th ed. Philadelphia: Lippincott Williams et Wilkins;2015.p.566-76

[3] Rosai J. Adrenal gland and other paraganglia. In: Rosai And Ackerman's Surgical Pathology. 10th ed. Philadelphia: Saunders Elsevier Inc; 2011.p.1068-75

[4] Shimada H, Nakagawa A, Pathology of the Peripheral Neuroblastic Tumours, Laboratory Medicine,2006;37:684-9.

[5] Fredlund E, Ringner M, Maris JM, Pahlman S. High Myc pathway activity and low stage of neuronal differentiation associate with poor outcome in neuroblastoma. Proc Natl Acad Sci U S A. 2008; 105:14094-9

[6] Maris J M. Recent Advances in Neuroblastoma. N Engl J Med. 2010; 362: 2202-11.

[7] Kumar V, Abbas KA, Aster JC. Tumors and tumor-like lesions of infancy and childhood. In: Robbins and Cotran Pathologic Basis of Disease. 9th ed. India: Elsevier; 2014.p. 475-9.

[8] Ambros IM, Speleman F, Ambros PF. Nervous system: Peripheral neuroblastic tumours (Neuroblastoma, 
Ganglioneuroblastoma, Ganglioneuroma). Atlas Genet Cytogenet Oncol Haematol.2009; 13:84-9.

[9] Ambros PF, Ambros IM, Brodeur GM, Haber M, Khan J, Nakagawara A et al. International consensus for neuroblastoma molecular diagnostics: Report from International Neuroblastoma Risk group (INRG) Biology Committee. Br J Cancer 2009; 100:1471-82.

[10] Franks LM, Bollen A, Seeger RC, Stram DO, Matthay KK. Neuroblastoma in adults and adolescents: an indolent course with poor survival.Cancer1997; 79: 2028-35.

[11] Lonergan GJ, Schwab CM, Suarez ES Carlson CL. Neuroblastoma, ganglioneuroblastoma, and ganglioneuroma: radiologic-pathologic correlation. Radiographics 2002; 22: 911-34.

[12] Asada Y, Marutsuka K, Mitsukawa T, Kuribayashi T,Taniguchi S Sumiyoshi A. Ganglioneuroblastoma of the thymus: an adult case with the syndrome of inappropriate secretion of antidiuretic hormone. Hum Pathol1996;27: 506-9.

[13] Adam A, Hochhholzer L. Ganglioneuroblastoma of the posterior mediastinum: A clinicopathologic review of 80 cases. Cancer 1981;47:373-81.

[14] Moukheiber AK, Nicollas R, Roman S, Coze C, Triglia JM. Primary pediatricneuroblastic tumours of the neck. Int J Pediatr Otorhinolaryngol 2001;60:155-61.

[15] Kleihues P. Pathology and Genetics of Tumours of the Nervous System. World Health Organization Classification of Tumours. Lyon: IARC Press; 2000. p. 141-4.

[16] Singh SK, Srivastava C, Ojha BK, Chandra A, Hussain N. Ganglioneuroblastoma of the conus: A rare and aggressive tumour. Neurol India 2010;58:966-8.
[17] Tripathy K, Misra A, Gouda AK, Das S, Das B. Primary intraspinalganglioneuroblastoma of the thoracic spine:A rare case report. Indian J PatholMicrobiol 2012; 55:5357.

[18] Moukheiber AK, Nicollas R, Roman S, Coze C, Triglia JM. Primary pediatricneuroblastictumours of the neck. Int J Pediatr Otorhinolaryngol 2001;60:155-61.

[19] Goto S, Umehara S, Gerbing RB, Histopathology (International Neuroblastoma Pathology Classification) and MYCN status in patients with peripheral neuroblastictumours: a report from the Children's Cancer Group. Cancer 2001; 92: 2699-708.

[20] Shimada H, Ambros IM, Dehner LP, Hata J, Joshi VV, Roald B. Terminology and morphologic criteria of neuroblastictumours: recommendations by the International Neuroblastoma Pathology Committee. Cancer 1999;86:349-63.

[21] Shawa H, Elsayes KM, Javadi S, Morani A, Williams MD, Lee JE. Adrenal ganglioneuroma: features and outcomes of 27 cases at referral cancer centre. Clinical Endocrinology 2014;80:342-7

[22] Kamińska E, Obołończyk L, Kmieć P, Czapiewski P, Babińska , Sworczak K. Report on 5 cases of adrenal ganglioneuromas. Acta Endocrinologica (Buc)2014;10: 477-86

[23] Louis CU, Shohet JM, Neuroblastoma: Molecular Pathogenesis and Therapy. Annu Rev Med. 2015; 66: 49-63.

[24] Shimada H, Umehara S. Monobe Y, Hachitanda Y, Nakagawa A, Goto S. International Neuroblastoma Pathology Classification for Prognostic Evaluation of Patients with Peripheral Neuroblastic Tumours A Report from the Children's Cancer Group.Cancer2001;92:245161 . 